\title{
Role of Transient Anions in Chemoradiation Therapy: Base Modifications, Crosslinks and Cluster Damages Induced to Cisplatin-DNA Complexes by 1-20 eV Electrons
}

Yanfang Dong, ${ }^{a}$ Yaxiao Wang, ${ }^{a}$ Puxiang Zhuang, ${ }^{a}$ Xianzhi Fu, ${ }^{a}$ Yi Zheng, ${ }^{a *}$ and Léon

\section{Sanche $^{b}$}

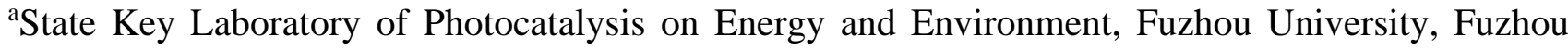
350116, P.R. China; ${ }^{b}$ Department of Nuclear Medicine and Radiobiology and Clinical Research Center, Faculty of Medicine, Université de Sherbrooke, Sherbrooke, QC Canada J1H 5N4

\section{RECEIVED DATE:}

TITLE RUNNING HEAD: clustered damages of cisplatin-DNA by LEEs

*CORRESPONDING AUTHOR: Yi Zheng

Fuzhou University, Fuzhou 350116, P.R. China.

Email address: Yizheng@fzu.edu.cn 


\section{Experimental Methods}

Platinating Plasmid DNA. Plasmid DNA (pGEM-3Zf(-), 3197 bp) was extracted from E. coli DH5 and purified with a HiSpeed plasmid Maxi kit (QIAGEN). The purified plasmid was dissolved in distilled deionized water $\left(\mathrm{ddH}_{2} \mathrm{O}\right)$ and consisted of $95.2 \%$ supercoiled, $0.4 \%$ crosslinks (CLs), 3.4\% single stand breaks (SSBs) and 1\% concatemeric forms. Cisplatin (cis-diamminedichloroplatinum(II), 99.9\% purity, Sigma-Aldrich) dissolved in $\mathrm{ddH}_{2} \mathrm{O}$ was mixed with the plasmid DNA solution in the initial molar ratios of 16.5 cisplatin per DNA, then incubated in the dark at room temperature for 2 hours. An efficiency of cisplatin binding to DNA of 33\% was measured by inductively-coupled-plasma mass spectroscopy. A mixed solution of unreacted cisplatin, TE buffer and cisplatin-DNA complexes were separated by gel filtration with a Sephadex G-50 medium. The molar ratio of platinum to DNA was found to be 5:1 on average in the cisplatin-DNA complexes.

LEE irradiation. The cisplatin-DNA samples (5 cisplatin molecules per DNA) were deposited on substrates consisting of $450 \pm 50 \mathrm{~nm}$ films of Ta evaporated onto a $0.4 \mathrm{~mm}$ thick silicon wafer. Seven $\mu \mathrm{l}$ of cisplatin-DNA solution containing of $320 \mathrm{ng}$ of DNA, were deposited onto the clean Ta surface. The deposited cisplatin-DNA samples were frozen with liquid nitrogen at $-65^{\circ} \mathrm{C}$ for 7 minutes in a glove box containing an atmosphere of pure dry nitrogen, the samples were lyophilized (freeze-dried) under a pressure of 7 mTorr by a hydrocarbon-free molecular-sieve pump for 2 hours. Assuming a density of DNA ${ }^{1}$ of $1.7 \mathrm{~g} \mathrm{~cm}^{-3}$ and deposited molecules to be uniformly distributed across the surface within a radius of $2 \pm 0.5 \mathrm{~mm}$, the average thickness of the film was estimated to be $15 \mathrm{~nm}$ (5 monolayers (ML)). Such a thickness has been widely used in DNA-LEE experiments. ${ }^{2}$

1 Fashman, G. D. Handbook of Biochemistry and Molecular Biology; CRC Press: Boca Raton, FL, 1995. 
The freeze-dried samples were transferred directly from the glove box under pure nitrogen to the LEE irradiation chamber also filled with pure nitrogen, which was subsequently evacuated for $\sim 24$ $\mathrm{h}$ to reach a base pressure of $2 \times 10^{-8}$ torr at ambient temperature. By changing the potential between the substrate (ground) and the center of the filament of the LEE gun, incident electron energy can be varied continuously from 1.4 to $20.4 \mathrm{eV}$. Taking as the zero electron-energy reference, the onset of electron transmission through the films, the latter range corresponds to absolute electron energies of 1 to $20 \pm 0.5 \mathrm{eV} .^{3}$ The film was bombarded with a $3 \mathrm{nA}$ incident electron beam at $1 \mathrm{eV}$, while in the range $2-20 \mathrm{eV}$, this current was $6 \mathrm{nA}$. Irradiation times at intervals of $1 \mathrm{eV}$ from 1 to $20 \mathrm{eV}$ were 5, 7, $10,15,20$ and 30s. The full width at half maximum of the electron beam was $\pm 0.3 \mathrm{eV}$. After an irradiation, the cisplatin-DNA samples were retrieved with $20 \mu \mathrm{l}$ of $\mathrm{ddH}_{2} \mathrm{O}$ with $95 \%$ efficiency. The cisplatin-DNA solution was divided into three identical portions: one for no enzyme treatment, the other two for additional enzyme treatment.

Enzyme treatment. Unirradiated (i.e., control) and irradiated cisplatin-DNA samples were treated with E.Coli base excision repair endonuclease III (Nth) and formamidopyrimidine $\mathrm{N}$ glycosylase (Fpg) (Trevigen Inc.). According to the linear portion of the enzyme response curves for strand breaks, the sensitivity of the enzyme to DNA was optimized at one unit of Fpg and 0.5 unit of Nth. These quantities were mixed with DNA after LEE radiation and incubated at $37^{\circ} \mathrm{C}$ for 60 min. One unit is defined as the cleavage at an AP site of a 34-bp oligonucleotide at a rate of $1 \mathrm{pmol} / \mathrm{h}$. The reactions were stopped by addition of $0.5 \mathrm{M}$ EDTA buffer and kept on ice prior to the further analysis of DNA damages.

2 Sanche, L. Low-Energy Electron Interaction with DNA: Bond Dissociation and Formation of Transient Anions, Radicals, and Radical Anions. In Radical and Radical Ion Reactivity in Nucleic Acid Chemistry; Greenberg, M. Ed.; Wiley: Hoboken, NJ, USA. 2009, pp 239-295.

3 Bao, Q.; Chen, Y.; Zheng, Y.; Sanche, L. Cisplatin Radiosensitization of DNA Irradiated with 2-20 eV Electrons: Role of Transient Anions. J. Phys. Chem. C. 2014, 118, 15516-15524. 
To reveal any heat labile effects, cisplatin-DNA untreated with enzymes was also kept at $37^{\circ} \mathrm{C}$ for $60 \mathrm{~min}$.

Quantification of DNA conformation variations. The different structural forms of DNA including supercoiled, nicked circular (SSB), linear (double strand break (DSB)) and CLs in each cisplatin-DNA sample were analyzed by running the samples on $1 \%$ agarose gel in $1 \mathrm{X}$ TAE buffer at 100 volts for 7 minutes, followed by 75 volts for 90 minutes. The samples and the agarose gels were stained with SYBR Green I at concentrations of 100X and 10000X, respectively. To obtained better accuracy, the binding efficiencies of SYBR Green I for the same amount (80 ng) of supercoiled and linear DNA was measured to determine a correction factor. This factor arises from the weaker binding of SYBR Green I to supercoiled DNA than to the nicked circular and linear forms. A factor of 1.69 was applied to the quantification of the different structural forms of DNA. The different DNA bands on the gel were analyzed by the ImageQuant 5.0 (Molecular Dynamics) software.

\section{Effective yields for DNA damages and the formation of specific lesions. The} percentage in the bombarded sample of CLs, SSBs and DSBs with or without enzyme treatment was measured as a function of electron fluence for each energy. The yields were obtained from the slope of these fluence-response curves at zero fluence. The experimental curves were fitted with a saturating exponential function, except for DSBs, which were fitted with a linear function. Fig. 1s shows an example of such a curve ( $\mathbf{a})$ obtained from the percentage in the sample of the SSB configuration as a function of fluence of $10 \mathrm{eV}$ electrons. The percentage yields at zero fluence are cause by all the manipulations of the samples. These damages do not influence the slope at zero fluence, which consequently represent only additional damages caused by LEE irradiation. The curves after treatment of Nth $(\Delta)$ and Fpg $(\bullet)$ enzymes are also displayed. Each data point in a fluence-response curve is the 
result of 8 identical measurements at a given fluence. Such curves were plotted for each type of conformational damage with and without enzyme treatment, for a total of 180 data points. Since the yields were obtained at 20 different energies, 3600 data points were needed to generate the yield functions presented in this article, if we include the control samples. Since the cross reactivity of Nth and Fpg for enzyme-sensitive sites is low, the enzyme-sensitive sites (ess) recognized by both enzymes are assumed independent. ${ }^{4}$ The yield of base damage was obtained from the difference between yields in the presence and absence of enzymatic treatment of irradiated DNA. The total yields of base lesions are obtained as follows:

$$
\mathrm{Y}(\text { base lesion })_{\mathrm{ess}}=\mathrm{Y}(\mathrm{Nth})+\mathrm{Y}(\mathrm{Fpg})-2[\mathrm{Y}(\mathrm{DNA})+\mathrm{Y}(\text { heat })]
$$

$\mathrm{Y}(\mathrm{Nth})$ and $\mathrm{Y}(\mathrm{Fpg})$ are defined as the yields of DNA damage from exposure-response curves with Nth or Fpg treatment. Y(DNA) indicates the yields of the prompt DNA damages without enzyme treatments. Y(heat) is the yield of heat liable sites that arose after the DNA samples were incubated at $37^{\circ} \mathrm{C}$ for $60 \mathrm{~min}$ without enzyme. The total yields of DNA damages are calculated as

$$
\mathrm{Y}(\text { total })=\mathrm{Y}(\mathrm{Nth})+\mathrm{Y}(\mathrm{Fpg})-\mathrm{Y}(\mathrm{DNA})-2 \mathrm{Y}(\text { heat })
$$

Fig. 2s-5s shown the effective yields of loss of supercoiled, SSBs, DSBs and CLs as a function of electron energy for cisplatin-DNA complexes with and without Fpg and Nth treatment.

4 Yokoya, A.; Cunniffe, S. M. T.; O'Neill, P. Effect of Hydration on the Induction of Strand Breaks and Base Lesions in Plasmid DNA Films by Gamma-Radiation. J. Am. Chem. Soc. 2002, 124, 8859-8866. 
Fig. 1s Exposure-response curves for SSBs in 5 ML 5:1 cisplatin-DNA films ( $\boldsymbol{\square}$ ) induced by $10 \mathrm{eV}$ electrons together with the parallel treatment with Fpg $(\bigcirc)$ and $\mathrm{Nth}(\boldsymbol{\Delta})$ enzymes. The dashed lines are exponential fits and the solid lines the initial slopes. Each data point is the result of eight identical experiments.

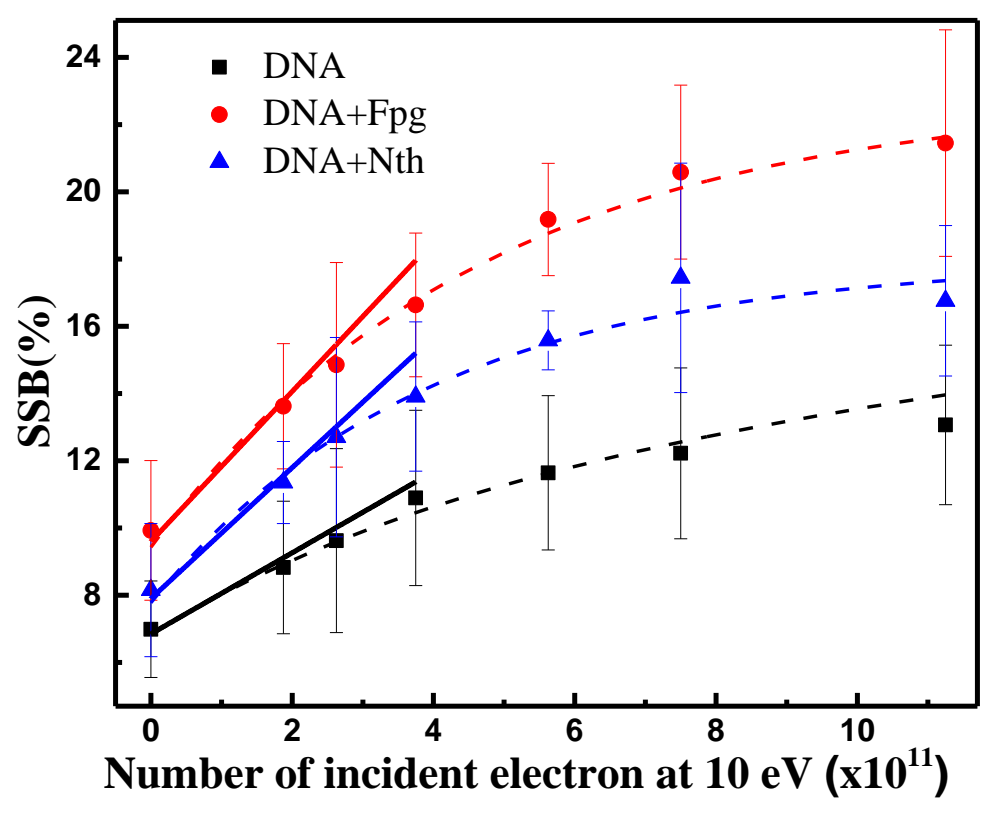


Fig. 2s A): Effective yields of loss of DNA supercoiled configuration induced by 1-20 eV electron impact on cisplatin-DNA films of 5 ML thickness, without enzymatic treatment ( $\mathbf{a})$ and with treatment by Fpg $(\bullet)$ and Nth $(\boldsymbol{\Delta})$. Each data point is the result of 8 identical experiments. The lines are guides to the eye.

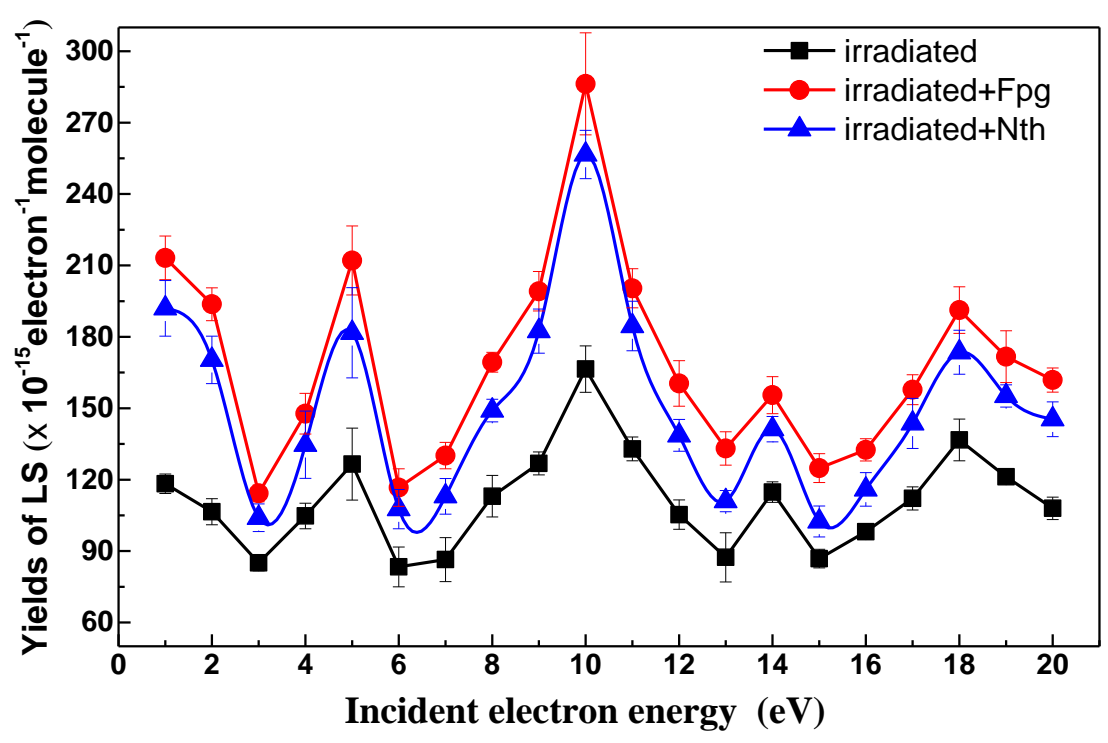


Fig. 3s A): Effective yields of formation of single strand breaks (SSBs) induced by 1-20 eV electron impact on cisplatin-DNA films of 5 ML thickness, without enzymatic treatment ( $\mathbf{a})$ and with treatment by Fpg $(\bullet)$ and Nth $(\boldsymbol{\Delta})$. Each data point is the result of 8 identical experiments. The lines are guides to the eye.

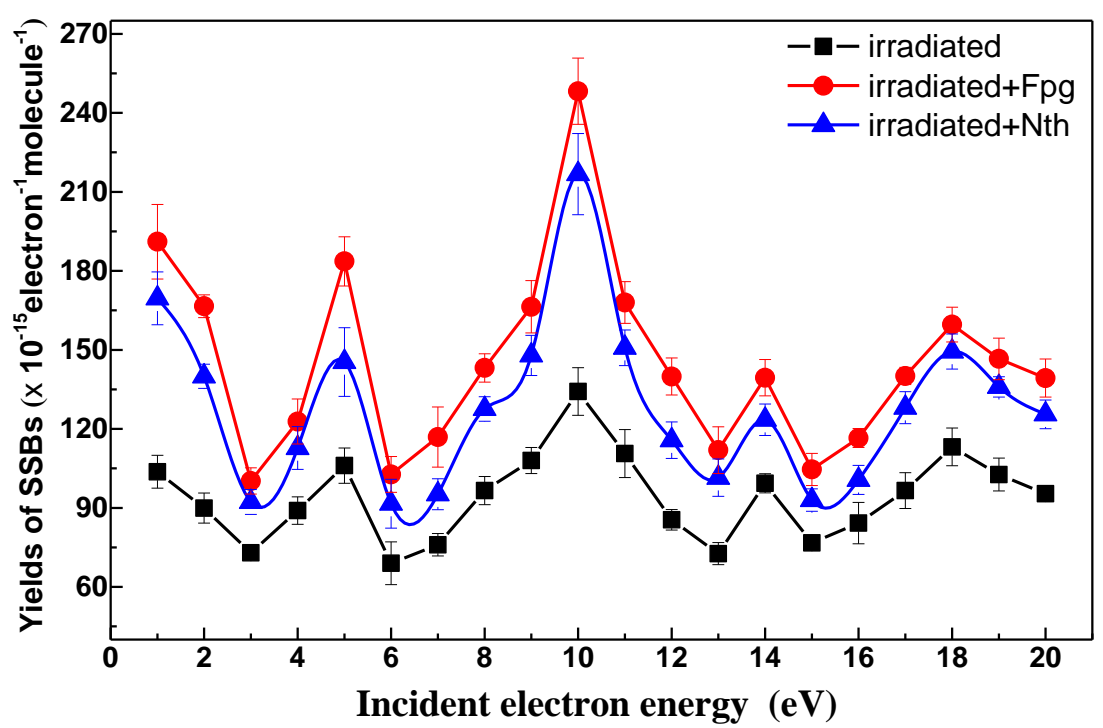


Fig. 4s A): Effective yields of formation of double strand breaks (DSBs) induced by 1-20 eV electron impact on cisplatin-DNA films of 5 ML thickness, without enzymatic treatment ( $\mathbf{a})$ and with treatment by Fpg $(\bullet)$ and Nth $(\boldsymbol{\Delta})$. Each data point is the result of 8 identical experiments. The lines are guides to the eye.

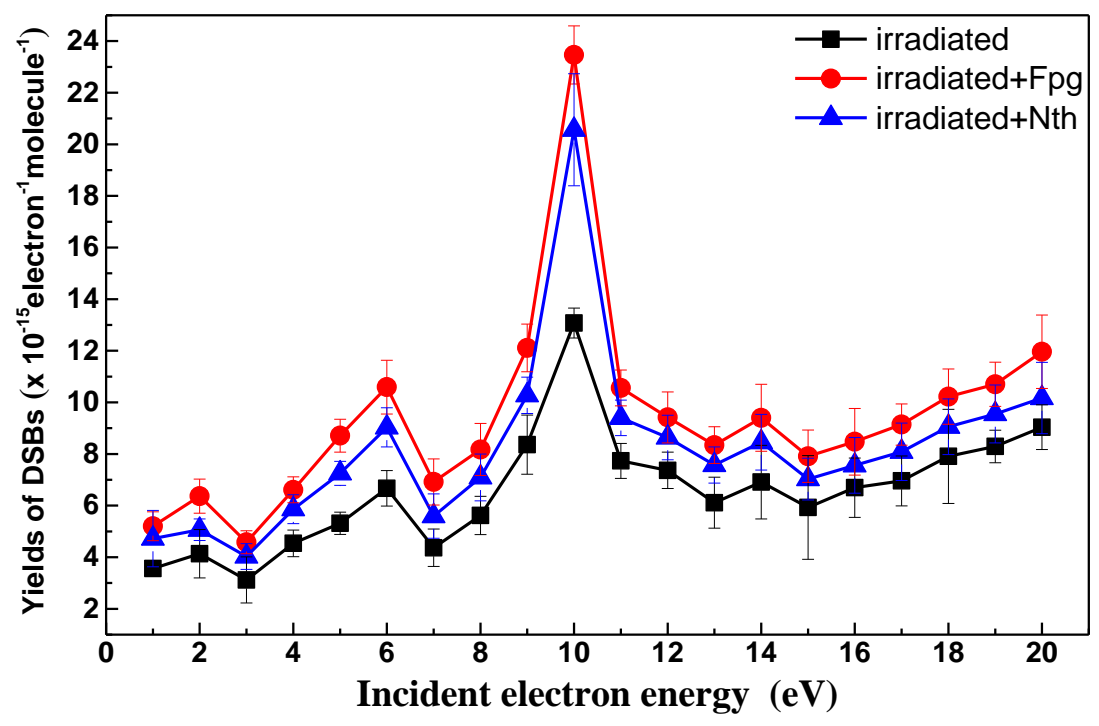


Fig. 5s A): Effective yields of formation of crosslinks induced by 1-20 eV electron impact on cisplatin-

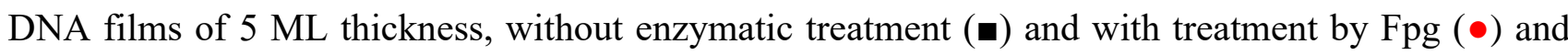
Nth $(\Delta)$. Each data point is the result of 8 identical experiments. The lines are guides to the eye.

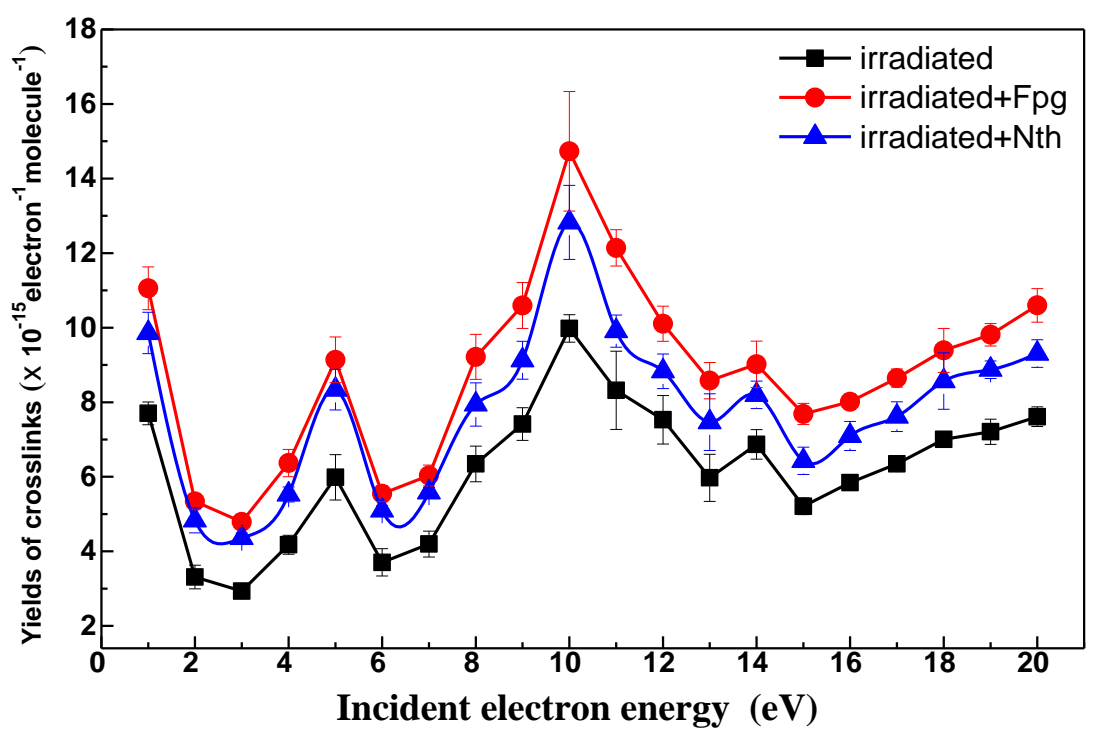

